\title{
Pulmonary complications following hematopoietic stem cell transplantation in children
}

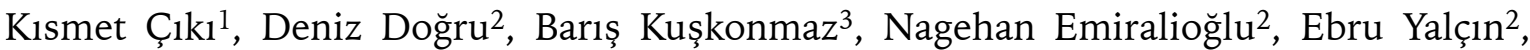 \\ Uğur Özçelik², Duygu Uçkan-Çetinkaya ${ }^{3}$, Nural Kiper ${ }^{2}$ \\ Departments of ${ }^{1}$ Pediatrics, ${ }^{2}$ Pediatric Pulmonology and ${ }^{3}$ Bone Marrow Transplantation Unit, Hacettepe University Faculty \\ of Medicine, Ankara, Turkey. E-mail: drnagehan@yahoo.com \\ Received:24th April 2018, Revised: 24th May 2018, Accepted: 4th June 2018
}

SUMMARY: Çıkı K, Doğru D, Kuşkonmaz B, Emiralioğlu N, Yalçın E, Özçelik U, Uçkan-Çetinkaya D, Kiper N. Pulmonary complications following hematopoietic stem cell transplantation in children. Turk J Pediatr 2019; 61: 59-70.

Pediatric data about early or long-term pulmonary complications of hematopoietic stem cell transplantation (HSCT) are limited. Here we aimed to evaluate children who were treated with HSCT in the last 10 years and developed pulmonary complications following HSCT and to determine their risk factors associated with pulmonary complications.

In this retrospective study, we evaluated 195 patients for the development of pulmonary complications after HSCT within a 10 years of period. Pulmonary complications developed in $71(36.4 \%)$ patients. Of the 71 patients who had pulmonary complications, 60 had one pulmonary complication, 11 had two pulmonary complications. Pulmonary complications were diagnosed as early in $42(51.2 \%)$ and late in $40(48.8 \%)$ episodes. Pulmonary complications were infectious in $28(34.1 \%)$, noninfectious in $20(24.4 \%)$ and both infectious and nonfectious in $34(41.5 \%)$ episodes. Pulmonary complications developed significantly more frequently in patients with malignancy, congenital immune deficiency and with at least one pulmonary disease before HSCT. The number of patients who had myeloablative conditioning regimen was significantly higher in the group of patients without pulmonary complications than the group with pulmonary complications. However, in multivariate analysis, none of these variables were shown to be effective in predicting pulmonary complications after HSCT $(\mathrm{p}>0.05)$. During follow up, 54 (65.8\%) episodes recovered, 20 (24.3\%) episodes resulted with death due to pulmonary complications, 6 (7.3\%) episodes had chronic pulmonary disease (bronchiolitis obliterans (BO) and bronchiolitis obliterans organizing pneumonia (BOOP)); 2 patients (each patient with one episode) were lost to follow up. In conclusion; identifying children who are at risk for severe pulmonary complications and close longitudinal follow-up after HSCT by pediatric pulmonologists is mandatory to increase survival and life quality of these patients.

Key words: hematopoietic stem cell transplantation, pulmonary complications, infectious complications, non-infectious complications, pulmonary function tests.

Hematopoietic stem cell transplantation (HSCT) has been used with increasing frequency for the treatment of malignant and nonmalignant diseases. Pulmonary complications are among the most common serious sequelae of HSCT and can cause serious morbidity and mortality. They depend on multiple factors like pretransplant intensive chemotherapy combined with localized or total body irradiation and posttransplant infectious and noninfectious complications. ${ }^{1}$ In adults, the incidence of pulmonary complications and the mortality rate after HSCT were reported to be $40-60 \%$ and $30 \%$, respectively. ${ }^{2}$ Incidence of pulmonary complications in children has been reported to be $25 \%$ in a report. ${ }^{3}$ Although early or longterm pulmonary complications of HSCT are well-recognized in adults, the pediatric data 
is limited.

In this study, we aimed to evaluate children that who were treated with HSCT in the last 10 years and developed pulmonary complications following HSCT and to determine their risk factors associated with pulmonary complications.

\section{Material and Methods}

In this retrospective study, all children underwent HSCT between January 2005 and December 2015 in our hospital's Bone Marrow Transplantation Unit and developed pulmonary complications following HSCT were included.

All patients were hospitalized in single rooms with hepafilters throughout HSCT period. Patients received acyclovir, fluconazole and trimethoprim-sulfomethaxozole for prophylaxis against Herpes simplex and Varicella zoster viruses, fungal infection and infection with Pneumocystis jiroveci, respectively. Cytomegalovirus (CMV) infection was routinely monitored using real-time PCR assays. Broadspectrum antibiotic coverage was initiated at the first evidence of fever $\left(\operatorname{Tmax}>38^{\circ} \mathrm{C}\right)$. Intravenous immunoglobulin was administered weekly at a standard dose of $400 \mathrm{mg} / \mathrm{kg}$ from day -1 to day +21 and depending on IgG levels thereafter.

Patients who had any respiratory complaint after HSCT were seen and recorded by a pediatric pulmonologist in our hospital. Complete medical records of patients with pulmonary complications were retrospectively evaluated. Patients' ages, diagnosis, source of HSCT, donor type, conditioning regimen were recorded. HSCT indications were categorized in six groups as malignancy, bone marrow failure, hemoglobinopathy, immunodeficiency, hemophagocytic syndrome and neurometabolic disease. Acute graft-versus-host disease (GVHD) was diagnosed and graded according to the Seattle criteria and chronic GVHD was evaluated according to NIH criteria. ${ }^{4,5}$

Radiological, pathological and flexible bronchoscopy findings as well as microbiological results of patients were evaluated in order to define the type of pulmonary complications. "Pulmonary complication" was defined as development of one or more of the following: newly developed infiltration in chest $\mathrm{x}$-ray or chest computed tomography (CT), tachypnea, wheezing, respiratory distress, decreased respiratory sounds, crackles, ronchi, hemoptysis and hypoxia. Pulmonary complications occured within 100 days of HSCT were considered as "early pulmonary complication" and those after 100 days were considered as "late pulmonary complication". Pulmonary complications were considered as "infectious" if microorganism could be shown in bronchoalveolar lavage (BAL), fine needle aspiration biopsy, blood culture, sputum culture or if microorganisms could not be cultured but concomitant galactomannan, CMV PCR positivity, clinical suspicion (high fever, infectious involvement in chest X-ray or CT, cinically improvement with antibiotic, antifungal or viral treatment) were present. ${ }^{6}$ Echocardiography was performed in all patients who were suspected to have pulmonary hypertension or venooclusive disease.

Noninfectious complications were defined as follows ${ }^{6-8}$ :

- Upper airway complications: Severe mucosal damage/mucositis which lead to upper airway inflammation, laryngeal edema, dysphagia, odynophagia, or aspiration pneumonitis.

- Pulmonary edema: Acute onset dyspnoea, weight gain, bibasilar crackles, hypoxaemia, chest radiographs consistent with diffusely increased vascular marking.

- Pulmonary alveolar hemorrhage: Bleeding from endotracheal tube with infiltrate on chest X-ray, X-ray infiltrate with drop in hemoglobin or occult hemorrhage that was shown by hemosiderin containing alveolar macrophages in BAL or biopsy.

- Peri-engraftment respiratory distress syndrome: Hypoxia and respiratory distress, chest radiographic findings including diffuse edema and pulmonary infiltrates and in some cases pleural effusion.

- Acute respiratory distress syndrome (ARDS): Acute onset of respiratory symptoms, chest radiograph with bilateral infiltrates, partial pressure of oxygen $(\mathrm{PaO} 2)$ to fraction of inspired oxygen $(\mathrm{FiO} 2)$ ratio $(\mathrm{P} / \mathrm{F}$ ratio) $200 \mathrm{~mm} \mathrm{Hg}$ or less, no clinical evidence of left atrial hypertension

- Bronchiolitis obliterans syndrome (BOS): Obstructive lung defect characterized by 
dyspnea on exertion, cough, or wheezing, small fixed airway obstruction on PFTs, mosaic perfusion on high resolution chest CT.

- Bronchiolitis obliterans organizing pneumonia (BOOP): Fever, dyspnea, and a non- productive cough, with PFTs revealing a restrictive defect, HRCT reveals peripheral air space consolidation, with ground-glass and nodular opacities. The gold standard for diagnosis is lung biopsy showing small airway fibrosis and accompanying interstitial and alveolar inflammation. However, we were not able to do a biopsy, and we diagnosed this situation depending on chest CT findings revealing peripheral air space consolidation with ground-glass and nodular opacities. ${ }^{9}$

- "Undetermined": Dyspnea, hypoxia and pulmonary infiltrates accompanied by pleural effusion and/or ascites with no clinical and/or laboratory signs of infection which respond to diuretics and steroid.

\section{Evaluation of pulmonary function tests (PFT):}

PFTs were performed by spirometry in children 6 years and older in accordance with American Thoracic Society standards; forced vital capacity (FVC), forced expiratory volume in 1 second (FEV1) and forced mid-expiratory flow (FEF25\%-75\%) measurements were expressed in liters and referred to a healthy population as a percentage of predicted value. ${ }^{9}$ PFT which is part of our regular follow-up were routinely performed at least for once in the last month before HSCT. After HSCT, if the patients had no pulmonary problems, spirometry was performed yearly, but if patients had pulmonary problems they had spirometry every 3-6 months depending on the severity of their pulmonary problem. PFTs performed in the last month before the HSCT and the annual best PFT values after the HSCT were recorded. FVC, FEV1 and FEV1/ FVC were categorized as normal $(\geq 80 \%)$ or reduced $(<80 \%)$ and FEF25\%-75\% was categorized as normal $(>75 \%)$ or reduced $(\leq \% 75 \%)$.

Patients were treated with antibiotics antifungal and antiviral therapy if infectious pulmonary complication was suspected or demonstrated. Oxygen support was given in hypoxic patients and patients were intubated and mechanically ventilated for acute respiratory failure.

The present study was approved by the local institutional review board with number GO16/614-15.

\section{Statistical Methods:}

The research data was uploaded to the computer environment and evaluated using "SPSS (Statistical Package for Social Sciences) for Windows 22.0 (SPSS Inc, Chicago, IL)." Descriptive statistics were presented as mean \pm standard deviation, median (minimummaximum), frequency distribution and percentage. Pearson Chi-square test and Fisher's exact test were used to evaluate categorical variables. The normal distribution suitability of the variables was examined using visual (histogram and probability plots) and analytical methods (Kolmogorov-Smirnov / Shapiro-Wilk Test). For the variables which were determined as not meeting the normal distribution; Mann-Whitney U test was used for statistical significance between two independent groups, The Kruskal Wallis Test was used for statistical significance between three independent groups. Post-hoc bonferroni correction was performed to determine the source of the difference when a significant difference was found between the three independent groups. In multivariate analysis, independent predictors of post-HSCT pulmonary complications which determined in previous analyzes were estimated using logistic regression analysis. Hosmer-Lemeshow test was used for model adaptation. $\mathrm{P}$ values of 0.05 were considered statistically significant.

\section{Results}

During the period of observation, 195 patients were treated with HSCT and pulmonary complications following HSCT developed in 71 patients $(36.4 \%)$. The most common underlying disease as indication of HSCT in these patients was malignant diseases in 30 patients $(42.3 \%)$ acute lymphatic leukemia being the most common in 8 patients, followed by congenital immune deficiency disorders in 10 (14.1\%), bone marrow failure (Fanconi aplastic anemia, aplastic anemia) in 9 (12.6\%),neurometabolic diseases in 9 (12.6\%), thalassemia major in $7(9.8 \%)$, and hemophagocytic syndrome in $6(8.6 \%)$ patients. 
Among 195 patients treated with HSCT, 59 had any pulmonary disease previous to HSCT (pneumonia, astma, bronchiectasis). The number of patients who had a pulmonary disease before HSCT was 29 out of 71 patients $(40.8 \%)$, and 30 out of 124 patients $(24.2 \%)$ with and without pulmonary complications after HSCT, respectively, and the difference between these two groups was statistically significant (p: 0.015).

Among patients with pulmonary complications $67.6 \%$ were males and $32.4 \%$ were females. Demographic findings, type and source of HSCT and characteristics of donors and recepients in patients with pulmonary complications after HSCT are shown in Table I.

Of the 71 patients who had pulmonary complications, 60 had one pulmonary complication, 11 had two pulmonary complications. Therefore, pulmonary complications following HSCT were evaluated on these 82 episodes. The earliest pulmonary complication was on the same day immediately after the HSCT, and the latest pulmonary complication was 4.5 years after the HSCT. Pulmonary complications were diagnosed as early in $42(51.2 \%)$ and late in 40 (48.8 $\%)$ episodes. Pulmonary complications were infectious in $28(34.1 \%)$, noninfectious in 20 (24.4\%) and both infectious and noninfectious in $34(41.5 \%)$ episodes. Among the early complications, 8 (19.1\%) were infectious, 19 (45.2\%) were non-infectious, 15 (35.7\%) were both infectious and noninfectious, and among the late onset complications, 20 (50\%) were infectious, 1 (2.5\%) was noninfectious, $19(47.5 \%)$ were both infectious and noninfectious. The incidence of noninfectious complications was significantly higher in the early onset, whereas it was significantly lower in the late onset $(p<0.001)$. The median (minimum and maximum) time from HSCT to complications was 190 (7- 1440) days in patients with infectious complications, 11 (4-120) days in patients with noninfectious complications, and 93 (1-1020) days in

Table I. Demographic Findings, Type and Source of HSCT and Characteristics of Donors and Recepients in Patients With Pulmonary Complications After HSCT.

\begin{tabular}{lccc}
\hline Variables & $\begin{array}{c}\text { First HSCT } \\
\text { (n:63) }\end{array}$ & $\begin{array}{c}\text { Second HSCT } \\
\text { (n:7) }\end{array}$ & $\begin{array}{c}\text { Third HSCT } \\
\text { (n: } 1)\end{array}$ \\
\hline Median age of diagnosis in months & 36 & 30 & 4 \\
Median age at time of HSCT in months & 70 & 66 & 18 \\
Median duration between diagnosis of underlying disease & 9 & 47 & 14 \\
and HSCT in months & 23 & 66 & 24 \\
Median Duration of follow-up after HSCT in months & $\mathrm{n}$ & $\mathrm{n}$ & $\mathrm{n}$ \\
Type of HSCT & 70 & 7 & 1 \\
Allogenic & 64 & 7 & 1 \\
HLA matched & 6 & 0 & \\
HLA mismatched & 1 & 0 & \\
Autologous & & & \\
Stem cell sources & 53 & 2 & \\
Bone marrow & 15 & 5 & \\
Peripheric blood & 3 & 0 & \\
Bone marrow + Cord blood & & & \\
Recipient- donor pairs & 11 & - & \\
Female- female & 12 & 1 & \\
Female- male & 24 & 5 & \\
Male- female & 24 & 1 & \\
Male-male & & & \\
\hline
\end{tabular}

HSCT, Hematopoietic stem cell transplantation 
patients with both infectious and noninfectious complications; this difference between these periods was statistically significant $(\mathrm{p}<0.001$; Kruskal Wallis Test) and in post-hoc binary comparison it was found that this significant difference was because of noninfectious complications which were seen in earlier period than the other complications

\section{Infectious Complications:}

In infectious pulmonary complication episodes, microorganisms could be detected in only 20 $(32.2 \%)$ episodes. Fine-needle aspiration biopsy was performed in one patient who had nodular lesions demonstrated in CT and Aspergillus fumigatus grew in tissue culture. Nine patients underwent fiberoptic flexible bronchoscopy and 7 BAL samples revealed 13 microorganisms (Aspergillus fumigatus, Pseudomonas aeruginosa, Klebsiella pneumonia, Gram positive bacilli, CMV, Parainfluenza). Candida albicans, E.coli and Acinetobacter baumani were grown in blood, Candida albicans, Aspergillus fumigatus were grown in sputum and Stenotrophomonas maltophilia and Klebsiella oxyto were grown in tracheal aspirate cultures

\section{Noninfectious pulmonary complications:}

Noninfectious complications were pleural effusionin $6(7.3 \%)$, pulmonary edema in $5(6.1 \%)$, BOS in $5(6.1 \%)$, upper airway complications in $3(3.7 \%)$, pre-engraftment respiratory distress syndrome in $3(3.7 \%)$, pulmonary alveolar hemorrhage in $(3.7 \%)$, ARDS in $2(2.4 \%)$, pneumomediastinum in $1(1.2 \%)$, and BOOP in $1(1.2 \%)$ patient. We could not define the disorder in 13 $(15.8 \%)$ patients and labelled the situation as "undetermined" which is described in Material Method section.

When early and late pulmonary complications were compared, it was found that the age of diagnosis of underlying disease and age at first HSCT were significantly younger in patients with early pulmonary complications $(\mathrm{p}<0.05)$. Among patients who had bone marrow as a source of HSCT, $34(81 \%)$ and $27(67.5 \%)$ had early and late complications, respectively; however patients who had peripheric stem cell as a source of HSCT had $5(11.9 \%)$ and $13(32.5 \%)$ early and late complications, respectively which was statistically significant.
Otherwise, there was no statistically significant difference between patients who had early versus late pulmonary complications according to type of HSCT (Allogenic - autologous), donor sex and recipient- donor sex and between patients who had myeloablative and nonmyeloablative regimens. On the other hand, when complications were evaluated according to the infections, it was found that patients who were younger at the time of the diagnosis of the underlying disease and at the time of first HSCT had more noninfectious complications. In all three groups ("infectious", "noninfectious", "both infectious and noninfectious"), number of patients who had myeloablative regimen and who had bone marrow as a source of stem cell was significantly higher (Table II).

\section{Risk factors for pulmonary complications:}

In 71 of the 82 complication episodes, patients were receiving immunosuppressive therapy; 37 patients had neutropenia, 31 patients had GVHD (15 of them were acute GVHD, 16 were chronic). When patients who developed pulmonary complications and the patients who had no pulmonary complications were compared, there was no statistically significant difference between type of HSCT, type of stem cell, and receipent- donor pairs, both after the first and the second HSCTs. Pulmonary complications developed significantly more frequently in patients with malignancy, congenital immune deficiency and with at least one pulmonary disease before HSCT. However, the number of patients who had myeloablative conditioning regimen was significantly higher in the group of patients without pulmonary complications than the group with pulmonary complications (Table III). However, in multivariate analysis, none of these variables were shown to be effective in predicting pulmonary complications after HSCT $(p>0.05)$.

\section{Pulmonary function tests:}

Only 29 patients had PFT with spirometry one month before HSCT and 11 of these patients had pulmonary complications after HSCT. There was no significant difference in pretransplant mean FEV1\%, FVC\%, FEV1/ FVC\% and FEF25-75\% values between patients with and without pulmonary complications. In the pretransplant PFTs, decreased FEV1 \% was 


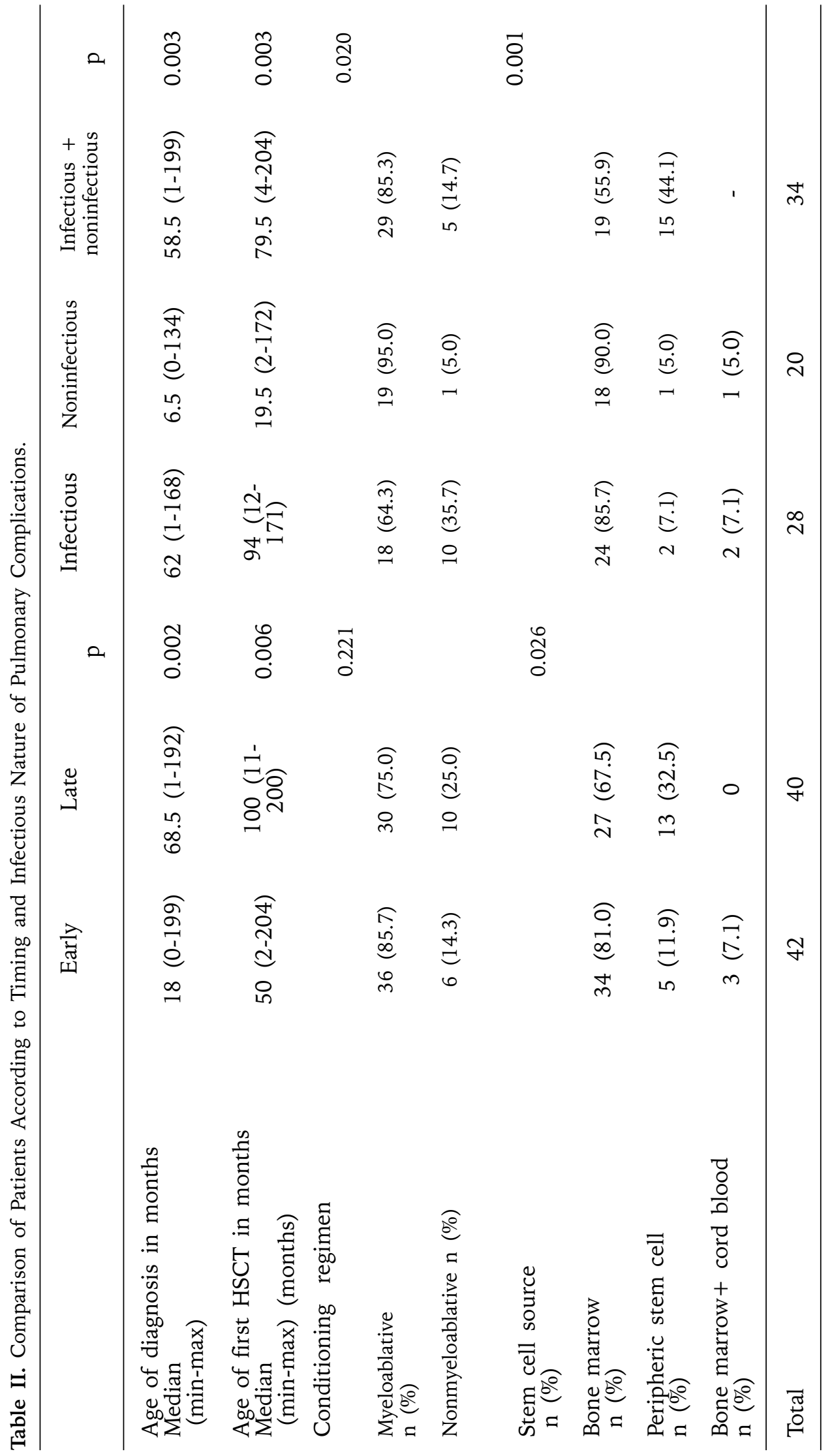


Table III. Comparison of Patients With Pulmonary Complication and Without Pulmonary Complication After HSCT.

\begin{tabular}{lccc}
\hline & $\begin{array}{c}\text { Patients with } \\
\text { pulmonary } \\
\text { complication } \\
\text { n (\%) } \\
\text { (Total n:71) }\end{array}$ & $\begin{array}{c}\text { Patients without } \\
\text { pulmonary } \\
\text { complication } \\
\text { n (\%) } \\
\text { (Total n:124) }\end{array}$ & $\mathrm{p}$ \\
\hline Female/male & $23 / 48$ & $46 / 78$ & 0.509 \\
Number of HSCT & $64(\% 90)$ & $117(\% 94)$ & 0.306 \\
$\quad 1$ & $7(\% 10)$ & $6(\% 5)$ & \\
$\quad 2$ & - & $1(\% 1)$ & \\
$\quad 3$ & $70(2-204)$ & $91.5(6-237)$ & 0.084 \\
Age of first HSCT in months & & & 0.002 \\
Median (min-max) & $9(1-181)$ & $20(1-231)$ & \\
Time between diagnosis and first HSCT & & & 0.002 \\
in months & $30(42.3)$ & $26(21.0)$ & 0.776 \\
Median (min-max) & $6(8.5)$ & $12(9.7)$ & 0.035 \\
HSCT indications & $7(9.9)$ & $27(21.8)$ & 0.002 \\
Malignancy & $9(12.7)$ & $40(32.3)$ & 0.044 \\
Hemophagocytic syndrome & $10(14.1)$ & $7(5.6)$ & 0.516 \\
Hemoglobinopathy & $9(12.7)$ & $12(9.7)$ & 0.015 \\
Bone marrow failure & $29(40.8)$ & $30(24.2)$ & 0.015 \\
Immunodeficiency & & & \\
Neurometabolic disease & $59(83.1)$ & $41(66.9)$ & \\
Previous history of pulmonary disease & $12(16.9)$ & & \\
Conditioning regimen & & & \\
Myeloablative & & & \\
Non-myeloablative & & & \\
\hline
\end{tabular}

HSCT: Hematopoietic stem cell transplantation

observed in 2 and 4 patients and decreased FVC\% was observed in 3 and 6 patients with pulmonary complications and without pulmonary complications, respectively.

In the 7-year follow-up after HSCT, median FEV $1 \%$ and FVC\% were statistically significantly lower in the first year in patients with pulmonary complications, and in the rest of the years this difference was not remarkable between two groups (Figs. 1 and 2). When PFTs of patients with and without GVHD who developed pulmonary complications were compared, although the number of patients are small, no statistically significant difference was noted between these two groups according to years after HSCT (Table IV).

\section{Treatment and Prognosis}

Median follow up period after HSCT were 41 months (minimum 1 month- maximum 137 months). During follow up, 54 (65.8\%) episodes recovered, $20(24.3 \%)$ episodes resulted with death due to pulmonary complications, 6 (7.3 $\%)$ episodes had chronic pulmonary disease (BOS and BOOP); 2 patients (each patient with one episode) were lost to follow up

Among 124 patients who had no pulmonary complications, $9(7.2 \%)$ patients died because of transplant related complications. Among 71 patients with pulmonary complications, death occurred in 18 patients $(25.3 \%)$ because of pulmonary complications; 4 of those patients had had a second HSCT. Among these 18 patients who died, 4 had relapse of their underlying diseases and therefore additional involvement of other systemic complications accompanying their cause of death, as well. Most common underlying disorder of patients with pulmonary complications who died was malignancy in 8 , bone marow failure in 3 , 


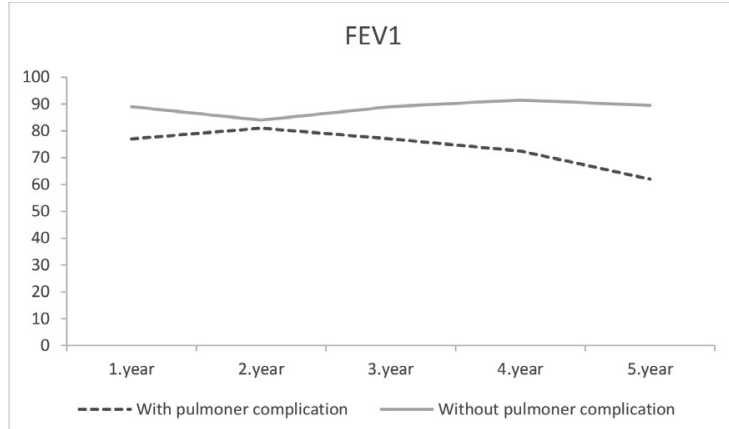

Fig. 1. Change of FEV1\% within years after transplantation

neurometabolic disease in 3 , hemophagocytic syndrome in 2 , hemoglobinopathy in 1 and congenital immune deficiency in 1 patient. Among 20 epsiodes which resulted with death, 11 (55\%) was early, $9(45 \%)$ was late; 3 (15\%) was infectious, $4(20 \%)$ was noninfectious, $13(65 \%)$ was both infectious and noninfectious; and the most common noninfectious complication was undetermined in 5 patients, followed by pleural effusion in 4, pulmonary hemorrhage in 3 , acute respiratory distress syndrome in 3 , upper airway complication in 1 and pneumomediastinum in 1 patient.

\section{Discussion}

In this retrospective report, we present data of children with pulmonary complications after HSCT in our center treated in 10 years. We showed that $36.4 \%$ of our patients developed a pulmonary complication after HSCT and $25.3 \%$ of these patients died because of pulmonary related complications. Pulmonary complications caused significant mortality in our patients with HSCT, only $7.2 \%$ of patients without pulmonary complications died because of other HSCT related complications. The incidence of pulmonary complications in the pediatric group in other reports are $25 \%$ and $74 \%$ in the literature 3,6 and in these reports, mortality because of a pulmonary complication was $47 \%$ which is significantly higher than our patients. ${ }^{6}$

In our study, we showed that pulmonary complications in the early period was slightly higher than the late period similar to the report by Kaya et al. ${ }^{6}$ who reported that infectious pulmonary complications and early complications were more frequently in children after HSCT.

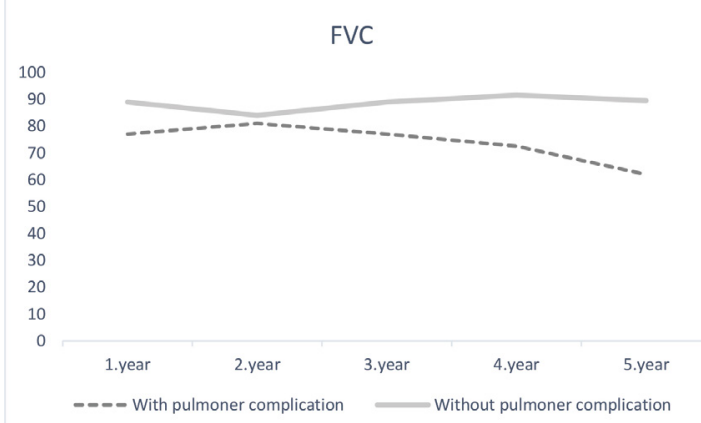

Fig. 2. Change of FVC\% within years after transplantation

Although non-infectious pulmonary complications are increasing in pediatric HSCT recipients, the majority of pulmonary complications are infectious ${ }^{10-12}$, similar to our study. Infectious complications are more common in allogeneic HSCT recipients because of GVHD itself and the prophylactic or therapeutic immunosuppressive therapy for GVHD. ${ }^{11}$

Evaluating for likely infectious pathogens is vital in any HSCT recipient with suggestive clinical findings. Flexible bronchoscopy with BAL is a valuable and safe method which is frequently used to evaluate infection in patients with pulmonary complications after HSCT and is favored over invasive methods like transbronchial biopsy and open lung biopsy because of the diagnostic yield and relatively low rate of complications. ${ }^{3}$ It has been shown to provide specific diagnoses in $50-94 \%$ of adult and pediatric BMT patients with pulmonary

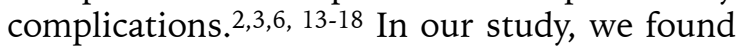
microbiologic evidence of infection in only 20 of 62 infectious episodes and the most pathogens were demonstrated from BAL. This low number can be attributed to the low number (only nine) of patients who underwent flexible bronchoscopy and BAL, because we could not perform bronchoscopy in most patients because of severe thrombocytopenia at the time of suspected pulmonary complication. In the British Thoracic Society guideline for adults, it is recommended that bronchoscopy with lavage can be performed with platelet counts $>20.000$ per $\mu \mathrm{L} .{ }^{19}$ Although we infused thrombocytes to patients with thrombocytopenia; we still could not perform bronchoscopy because of poor general status of some patients due to problems like deep hypoxia or cardiac problems. Additionally, in some patients, we postponed 
Table IV. Comparison of Pulmonary Function Test of Patients With and Without GVHD Who Developed Pulmonary Complications.

\begin{tabular}{|c|c|c|c|c|c|}
\hline \multirow[b]{3}{*}{ PFT } & \multicolumn{4}{|c|}{ GVHD } & \multirow[t]{3}{*}{$\mathrm{p}^{*}$} \\
\hline & \multicolumn{3}{|c|}{ Yes } & No & \\
\hline & $\mathrm{n}$ & Median & $\mathrm{n}$ & Median & \\
\hline 1 year after HSCT & 7 & & 16 & & \\
\hline FEV1 \% & & 76 & & 79.5 & 0.871 \\
\hline FVC\% & & 71 & & 78 & 0.671 \\
\hline FEV1/FVC\% & & 101 & & 101.5 & 0.492 \\
\hline $\mathrm{FEF}_{25-75 \%}$ & & 80 & & 95.5 & 0.624 \\
\hline 2 years after HSCT & 4 & & 9 & & \\
\hline FEV1\% & & 89.5 & & 85 & 0.710 \\
\hline $\mathrm{FVC} \%$ & & 89.5 & & 81 & 0.825 \\
\hline FEV1/FVC\% & & 98.5 & & 101 & 0.940 \\
\hline $\mathrm{FEF}_{25-75} \%$ & & 95 & & 89 & 0.710 \\
\hline 3 years after HSCT & 4 & & 5 & & \\
\hline FEV1\% & & 77 & & 78 & 0.730 \\
\hline FVC\% & & 72.5 & & 83 & 0.905 \\
\hline FEV1/FVC\% & & 94.5 & & 106 & 1.000 \\
\hline $\mathrm{FEF}_{25-75 \%}$ & & 102.5 & & 94 & 1.000 \\
\hline 4 years after HSCT & 2 & & 4 & & \\
\hline FEV $1 \%$ & & 67 & & 86 & ----- \\
\hline FVC\% & & 61 & & 78.5 & ----- \\
\hline FEV1/FVC\% & & 101.5 & & 106.5 & ----- \\
\hline $\mathrm{FEF}_{25-75 \%}$ & & 120.5 & & 118 & ----- \\
\hline 5 years after HSCT & 1 & & - & & \\
\hline FEV $1 \%$ & & 69 & & ----- & ----- \\
\hline FVC\% & & 62 & & ----- & ----- \\
\hline FEV1/FVC\% & & 108 & & ----- & ----- \\
\hline $\mathrm{FEF}_{25-75 \%}$ & & 98 & & ----- & ----- \\
\hline
\end{tabular}

${ }^{*}$ Mann-Whitney U test, HSCT: Hematopoietic stem cell transplantation

bronchoscopy as wide spectrum antibiotics were already started before bronchoscopy, which would prevent growth of microorganisms. Flexible bronchoscopy with BAL can also reveal primary or secondary malignancy, and is helpful in the diagnosis of alveolar hemorrhage and can lead to treatment change in patients. ${ }^{20,21}$

With the decrease of the incidence of infectious pulmonary complications because of effective prophylactic therapy, noninfectious pulmonary complications have emerged as a major cause of morbidity and mortality in patients treated with HSCT. ${ }^{11}$ Among non infectious complications, most of our patients presented with hypoxia and pulmonary infiltrates accompanied by pleural effusion and/or ascites with no clinical and/or laboratory signs of infection which responded to diuretics and steroid; we named this clinical situation as "undetermined". Actually, if we had done pulmonary biopsies, we might have had diagnosed these patients as pulmonary veno-occlusive disease (VOD), because some of these patients were diagnosed as hepatic VOD at the same time, as well, although we did not demonstrate pulmonary hypertension. Pulmonary VOD presents as a form of pulmonary hypertension, with dyspnea, signs of right-sided heart failure, and pulmonary infiltrates on chest radiographs. Hepatic VOD which is another vascular complication of HSCT is often associated with pulmonary VOD and interstitial pneumonitis. ${ }^{7,8}$

Our other common non-infectious pulmonary complications were pleural effusion, pulmonary edema, bronchiolitis obliterans. A diagnosis of bronchiolitis obliterans relies on histologic 
analysis of a surgical lung biopsy which shows an obliterative bronchiolitis characterized by thickening of the bronchiolar wall via inflammatory fibrosis. However, as biopsy is invasive and associated with risk of bleeding and other complications, experts now endorse the diagnosis of BOS using PFT. ${ }^{22}$ All of our patients were diagnosed with PFT and HRCT, therefore we named all of them as BOS.

Pulmonary involvement is common in chronic GVHD and it is well documented that BOS is associated with chronic GVHD. ${ }^{7}$ BOS is characterized by the new onset of an obstructive lung defect. Clinical manifestations may include dyspnea on exertion, cough or wheezing. Cryptogenic organizing pneumonia (formerly known as BOOP) and progressive restrictive lung disease in the absence of extrapulmonary causes are other entities that are not currently considered to be diagnostic or distinctive of lung chronic GVHD but remain areas of active investigation. ${ }^{22}$

In our study, we also demonstrated that noninfectious complications were statistically significant more often seen in the early period and statistically significantly more frequent in neurometabolic disorders. However, infectious complications were statistically more frequent in the bone marrow failure group.

HSCT indications, primary diagnosis, conditioning regimen, neutropenia, GVHD, previous pulmonary disease, donor type, gender difference between recipient and donor, duration between the diagnosis of the underlying disease and time of HSCT are risk factors for pulmonary complications after HSCT.7,8,23-25 In our study, although we could not find any factors which predict pulmonary complications after HSCT in multivariate analysis, pulmonary complications seemed to occur significantly more frequently in the malignancy and congenital immune deficiency groups. We also found that hemoglobinopathy and bone marrow deficiency patients developed significantly less pulmonary complications. This situation can be explained by the absence of previous lung disease in hemoglobinopathy and bone marrow failure group and immune dysregulation and previous pulmonary infections that can be seen in the natural course of patients with malignancy and congenital immunodeficiencies. We additionally found increased frequency of pulmonary complications in patients with previous lung disease before HSCT and in patients who received myeloablative conditioning regimen.

PFTs are used for diagnosis and follow-up of pulmonary complications after HSCT. Studies which report the predictive value of pretransplant PFTs for posttransplant complications suggest that poor lung function before transplant increases the risk of posttransplant complications. ${ }^{26}$ In our study, only 29 patients underwent PFT studies before HSCT. There was no significant difference in median spirometric values in the patients with and without pulmonary complications which may be attributed to the small number of patients. Kaya et al. also reported that pretransplant PFTs were not significantly correlated to the risk of pulmonary complications and overall survival, but patients with reduced pretransplant FEV1 or FVC were significantly more likely to develop early respiratory failure. ${ }^{6}$

Deterioration of pulmonary function after HSCT is a well-known effect of this treatment; BOS may be seen in patients with chronic GVHD and restrictive disorders are generally due to the pulmonary toxicity of chest irradiation and chemotherapy. ${ }^{27}$ In a study of pediatric patients, after HSCT restrictive and/or diffusion abnormalities were most prevalent during the first year after SCT, with improvement over time, but no normalization. ${ }^{28}$ As our study was retrospective, not all patients had PFTs following HSCT in a long period. Spirometric parameters (FEV1\% and $\mathrm{FVC} \%$ ) were statistically significantly lower in the first year in patients with pulmonary complications which was not remarkable. In the second year FEV $1 \%$ and FVC\% of patients with pulmonary complications improved. However our number of patients who underwent PFT after HSCT was not sufficient. Similarly, one study reported that there was a reduction in PFT after HSCT and improvement over time. ${ }^{29}$

Our study is the first in our country which evaluates pulmonary complications after HSCT in the pediatric age group. The main limitations are its being retrospective, the lack of transbronchial biopsies, low number of flexible bronchoscopies and BAL specimens and low number of patients who had spirometry before and after HSCT. The study would have been much better if we could have measured 
lung volumes by pletismography and lung diffusion in the long term follow up.

Pulmonary complications after HSCT most commonly occur and if not treated, they can progress and become irreversible in the long term and contribute significantly to morbidity and mortality. Identifying children who are at risk for severe pulmonary complications as early as possible and a close longitudinal followup after HSCT by pediatric pulmonologists is mandatory to increase survival and life quality of these patients. Based on our results, children with malignancy, congenital immune deficiency, previous lung disease and patients who receive myeloablative conditioning regimen are at higher risk for developing pulmonary complications after HSCT. It should always be kept in mind that some patients with pulmonary involvement can be asymptomatic early in the disease process. Therefore, all children that receive HSCT should have a baseline PFT before HSCT, and then, should have regular clinical visits including spirometry at the end of the first sixth month and the first year of HSCT and yearly thereafter and HRCT of thorax should be obtained if indicated. Earlier and more frequent clinical assessment including PFT's should be obtained in patients with chronic GVHD or in patients whose pulmonary symptoms and signs get worse over time. Further long term longitudinal studies with increased number of patients are definitely needed.

\section{REFERENCES}

1. Griese M, Rampf U, Hofmann D, et al. Pulmonary complications after bone marrow transplantation in children: Twenty-four years of experience in a single pediatric center. Pediatr Pulmonol 2000; 30: 393-401.

2. Kotloff RM, Ahya VN, Crawford SW. Pulmonary complications of solid organ and hematopoietic stem cell transplantation. Am J Respir Crit Care Med 2004; 170: 22-48.

3. Eikenberry M, Bartakova H, Defor T, et al. Natural history of pulmonary complications in children after bone marrow transplantation. Biol Blood Marrow Transplant 2005; 11: 56-64.

4. Thomas ED, Storb R, Clift RA, et al. Bone-marrow transplantation (second of two parts). N Engl J Med 1975; 292: 895-902.

5. Filipovich $\mathrm{AH}$, Weisdorf D, Pavletic S, et al. National Institutes of Health consensus development project on criteria for clinical trials in chronic graft-versus-host disease: I. Diagnosis and staging working group report. Biol Blood Marrow Transplant 2005; 11: 945-956.
6. Kaya Z, Weiner DJ, Yilmaz D, Rowan J, Goyal RK Lung function, pulmonary complications, and mortality after allogeneic blood and marrow transplantation in children. Biol Blood Marrow Transplant 2009; 15: 817-826.

7. Khurshid I, Anderson LC. Noninfectious pulmonary complications after bone marrow transplantation. Postgrad Med J 2002; 78: 257-262.

8. Michelson PH, Goyal R, Kurland G. Pulmonary complications of haematopoietic cell transplantation in children. Paediatric Respir Rev 2007; 8: 46-61.

9. Yanik G, Kitko C. Management of noninfectious lung injury following hematopoietic cell transplantation. Curr Opin Oncol 2013; 25: 187-194.

10. Standardization of Spirometry, 1994 Update. American Thoracic Society. Am J Respir Crit Care Med. 1995; 152: 1107-1136.

11. Lim DH, Lee J, Lee HG, et al. Pulmonary complications after hematopoietic stem cell transplantation. J Korean Med Sci 2006; 21: 406-411.

12. Srinivasan A, Srinivasan S, Sunthankar S, et al., PreHematopoietic Stem Cell transplant lung function and pulmonary complications in children. Ann Am Thorac Soc 2014; 11: 1576-1585.

13. McCubbin MM, Trigg ME, Hendricker CM, Wagener JS. Bronchoscopy with bronchoalveolar lavage in the evaluation of pulmonary complications of bone marrow transplantation in children. Pediatr Pulmonol 1992; 12: 43-47.

14. Lanino E, Sacco O, Kotitsa Z, et al. Fiberoptic bronchoscopy and bronchoalveolar lavage for the evaluation of pulmonary infiltrates after BMT in children. Bone Marrow Transplant 1996; 18: 117-120.

15. Ben Ari J, Yaniv I, Nahum E, Stein J, Samra Z, Schonfeld T. Yield of bronchoalveolar lavage in ventilated and nonventilated children after bone marrow transplantation. Bone Marrow Transplant 2001; 27: 191-194.

16. Bojko T, Notterman DA, Greenwald BM, De Bruin WJ, Magid MS, Godwin T. Acute hypoxemic respiratory failure in children following bone marrow transplantation: An outcome and pathologic study. Crit Care Med 1995; 23: 755-759.

17. Gilbert CR, Lerner A, Baram M, et al. Utility of flexible bronchoscopy in the evaluation of pulmonary infiltrates in the hematopoietic stem cell transplant population - a single center fourteen year experience. Arch Bronconeumol 2013; 49: 189-195.

18. Qualter E, Satwani P, Ricci A, et al. A Comparison of bronchoalveolar lavage versus lung biopsy in pediatric recipients after stem cell transplantation. Biol Blood Marrow Transplant 2014; 20: 1229-1237.

19. Du Rand IA, Blaikley J, Booton R, et al. British Thoracic Society guideline for diagnostic flexible bronchoscopy in adults: accredited by NICE. Thorax 2013; 68: i1-i44.

20. Srivastava A, Gottlieb D, Bradstock KF. Diffuse alveolar hemorrhage associated with microangiopathy after allogeneic bone marrow transplantation. Bone Marrow Transplant 1995; 15: 863- 867. 
21. Reichenberger F, Habicht J, Matt P, et al. Diagnostic yield of bronchoscopy in histologically proven invasive pulmonary aspergillosis. Bone Marrow Transplant 1999; 24: 1995-1999.

22. Jagasia MH, Greinix HT, Arora M, et al. National Institutes of Health Consensus Development Project on Criteria for Clinical Trials in Chronic Graft-versusHost Disease: I. The 2014 Diagnosis and Staging Working Group report. Biol Blood Marrow Transplant 2015; 21: 389-401.

23. Chi AK, Soubani AO, White AC, Miller KB. An update on pulmonary complications of hematopoietic stem cell transplantation. Chest 2013; 144: 1913-1922.

24. Pena E, Souza AC, Escuissato DL, et al., Noninfectious pulmonary complications after hematopoietic stem cell transplantation: practical approach to imaging diagnosis. Radiographics 2014; 34: 663-683.

25. Soubani AO, Pandya CM. The spectrum of noninfectious pulmonary complications following hematopoietic stem cell transplantation. Hematol Oncol Stem Cel Ther 2010; 3: 143-157.
26. Chien JW, Madtes DK, Clark JG. Pulmonary function testing prior to hematopoietic stem cell transplantation. Bone Marrow Transplant 2005; 35: 429-435.

27. Hoffmeister PA, Madtes DK, Storer BE, Sanders JE. Pulmonary function in long-term survivors of pediatric hematopoietic cell transplantation. Pediatr Blood Cancer 2006; 47: 594-606.

28. Wieringa J, Kralingen KW, Sont JK, Bresters D. Pulmonary function impairment in children following hematopoietic stem cell transplantation. Pediatr Blood Cancer 2005; 45: 318-323.

29. Ginsberg JP, Aplenc R, McDonough J, et al. Pretransplant lung function is predictive of survival following pediatric bone marrow transplantation. Pediatr Blood Cancer 2010; 54: 454-460. 